\section{FRI0045 CHANGES IN LEFT VENTRICULAR SYSTOLIC FUNCTION ARE PREDICTED BY DISEASE SEVERITY IN PATIENTS WITH RHEUMATOID ARTHRITISWITHOUT PRIOR CARDIOVASCULAR DISEASE}

Alessandro Giollo ${ }^{1}$, Giovanni Cioffi ${ }^{1}$, Giovanni Orsolini ${ }^{1}$, Luca Idolazzi ${ }^{1}$, Antonio Carletto ${ }^{1}$, Andrea Dalbeni ${ }^{2}$, Federica Ognibeni ${ }^{1}$, Elena Fracassi ${ }^{1}$, Davide Gatti ${ }^{1}$, Maurizio Rossini ${ }^{1}$, Ombretta Viapiana'. ' University of Verona, Rheumatology Unit, Department of Medicine, Verona, Italy; ${ }^{2}$ University of Verona, General Medicine and Hypertension Unit, Verona, Italy

Background: Occult left ventricular (LV) systolic dysfunction (LVSD) is associated with worse cardiovascular outcomes in asymptomatic patients with arterial hypertension and/or diabetes mellitus. In these patients, LV systolic function may worsen or improve overtime. However, little is known about the changes in LV function during follow up of patients with rheumatoid arthritis (RA).

Objectives: This prospective study analyzed incidence and factors associated with changes in LV systolic function in patients with RA without known cardiovascular disease.

Methods: One-hundred-forty outpatients with RA without overt cardiac disease were recruited between March and December 2014. Patients underwent clinical and echocardiographic evaluation at baseline and after a median period [min-max] of 35 [23-47] months of follow up. Stress-corrected midwall fractional shortening (sc-MFS) was used as measure of LVSD and considered impaired if $<86.5 \%$. Disease activity was assessed with the clinical disease activity index (CDAl).

Results: Impaired sc-MFS at follow up (sc-MFS-FU) was detected in 60/ $140(43 \%)$ patients who were compared with 80 patients with normal sc-MFS-FU. Baseline sc-MFS did not differ significantly between the two groups, which appeared to diverge significantly soon after 1-year of follow up (figure). Baseline anti-citrullinated protein antibodies (ACPA) positivity, high disease activity $(\mathrm{CDAl}>10)$ and duration of RA were independently associated with impaired sc-MFS-FU at multiple logistic regression analysis. Among these parameters, ACPA had the highest sensitivity $(80 \%)$ whereas high disease activity had the highest specificity $(89 \%)$ for sc-MFS-FU. A predictive score including all three predictors (ACPA status, CDAl>10 and duration of RA) detected impaired Sc-MFSFU with a sensitivity of $78 \%$ and specificity of $82 \%$ (AUC 0.80 [IC $0.72-0.88], p<0.0001$ )

Conclusion: This study supports the hypothesis that changes overtime in LVSF in patients with RA are associated with ACPA, high disease activity and duration of disease.

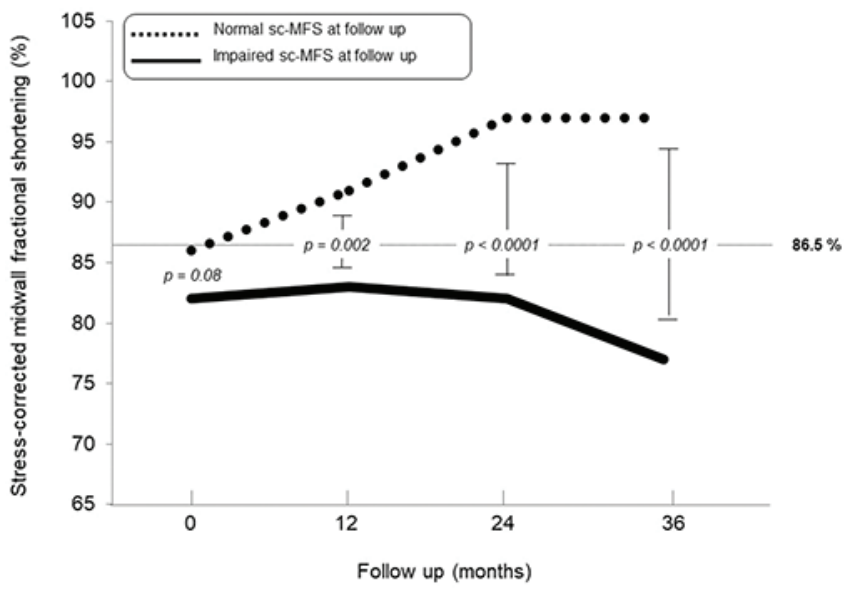

Disclosure of Interests: Alessandro Giollo: None declared, Giovanni Cioffi: None declared, Giovanni Orsolini Speakers bureau: Grunenthal, Luca Idolazzi: None declared, Antonio Carletto Speakers bureau: Roche, Novartis, MSD, Abbvie, Bristol, Jannsen, Celgene, Pfizer, Andrea Dalbeni: None declared, Federica Ognibeni: None declared, Elena Fracassi Speakers bureau: Novartis, Davide Gatti Speakers bureau: Abiogen, Amgen, Janssen-Cilag, Mundipharma, Pfeizer, Maurizio Rossini: None declared, Ombretta Viapiana Speakers bureau: Novartis, Abbvie, Eli-Lilly, Sanofi Genzyme DOI: 10.1136/annrheumdis-2019-eular.3459

\section{FRI0046}

POLYPHARMACY: A RARELY-DISCUSSED PROBLEM IN RHEUMATOID ARTHRITIS. DATA FROM A LARGE REAL-LIFE STUDY

Ana Paula Gomides ${ }^{1}$, Cleandro Albuquerque ${ }^{1}$, Ana Beatriz Vargas-Santos ${ }^{2}$, Claiton Brenol $^{3}$, Ivanio Pereira ${ }^{4}$, Karina Bonfiglioli ${ }^{5}$, Manoel Bertolo ${ }^{6}$, Maria Raquel Pinto ${ }^{7}$, Maria de Fátima Sauma ${ }^{8}$, Paulo Louzada $\mathrm{Jr}^{9}$, Rina Giorgi ${ }^{10}$, Sebastiao Radominski ${ }^{11}$, Licia Mota ${ }^{1}$, Geraldo Castelar ${ }^{2}{ }^{1}$ University of Brasilia, Brasilia, Brazil; ${ }^{2}$ UERJ, Rio de Janeiro, Brazil; ${ }^{3}$ Universidade Federal do Rio Grande do Sul, Porto Alegre, Brazil, ${ }^{4}$ Hospital Universitário - Universidade Federal de Santa Catarina, Florianópolis, Brazil; ${ }^{5}$ Hospital das Clinicas, Faculdade de Medicina da USP (HCFMUSP), São Paulo, Brazil; ${ }^{6}$ Faculdade de Ciências Médicas da Universidade Estadual de Campinas, Campinas, Brazil, ${ }^{7}$ Hospital das Clínicas da Universidade Federal de Minas Gerais, Belo Horizonte, Brazil; ${ }^{8}$ Faculdade de Medicina - Universidade Federal do Pará, Belém, Brazil;

${ }^{9}$ Faculdade de Medicina da Universidade de Ribeirão Preto, Ribeirão Preto, Brazil;

${ }^{10}$ Hospital do Servidor Público Estadual de São Paulo, São Paulo, Brazil;

${ }^{11}$ Universidade Federal do Paraná, Curitiba, Brazil

Background: Polypharmacy is concomitant use of five or more drugs and is usually associated with various comorbidities and advanced age. The already demonstrated consequences of polypharmacy are increased side effects, drug interactions, increased hospitalizations, drug handling difficulties, reduced adherence, and even increased mortality. Rheumatoid arthritis (RA) is a chronic systemic disease with preferential involvement between the third and fifth decades. Owing to treatment of underlying disease and comorbidities, patients with RA frequently require combined use of several drugs. Despite the possible negative consequences of polypharmacy in RA, there is a shortage of studies in literature.

Objectives: Evaluate prevalence and factors associated with polypharmacy in patients with RA in a real-life setting.

Methods: This is a cross-sectional part of the Rheumatoid Arthritis in Real Life (REAL) study that analyzed RA patients in a real-life context from August to October 2015 in 11 public health services in Brazil. Statistical analysis involved obtaining frequencies of the variables of interest and multiple Poisson regression analyses.

Results: We evaluated 792 patients, of whom $89 \%$ were women, with median age of 56.6 years. $78.73 \%$ had a positive rheumatoid factor and $55.2 \%$ had an erosive disease. The median Disease Activity Score was 3.52, the median CDAl score was 9 . Of the patients, $67.9 \%$ used 5 or more drugs as follows: mean of 2.8 and maximum of 5 drugs for RA, mean of 2.7 and maximum of 9 different types of medications for comorbidities, and mean of 5.5 and maximum of 11 drugs in total. The most commonly used drugs, besides specific drugs for underlying diseases, were vitamin D $(63.8 \%)$, folic acid $(61.1 \%)$, calcium $(57 \%)$, and antihypertensive drugs $(47 \%)$. In the multivariate analysis, the use of corticosteroids, methotrexate, and biological DMARDs and number of comorbidities (all with $\mathrm{P}<0.001$ ) were statistically significant. Age, schooling, rheumatoid factor positivity, and measures of activity by compound disease activity indexes were not associated with polypharmacy.

Conclusion: The prevalence of polypharmacy was high $(67.9 \%)$ in this population with RA, much higher than that described for elderly populations and other chronic diseases. This data can serve as an alert for this important and rarely-discussed aspect of RA.

\section{REFERENCES}

[1] Masnoon N, Shakib S, Kalisch-Ellett L, Caughey GE. What is polypharmacy? A systematic review of definitions. BMC Geriatrics 2017;17:230.

[2] da Rocha Castelar-Pinheiro G, Vargas-Santos $A B$, de Albuquerque $C P$, Bértolo MB, Júnior PL, Giorgi RDN, et al. The REAL study: a nationwide prospective study of rheumatoid arthritis in Brazil. Adv Rheumatol 2018;58:9.

[3] Kim J, Parish AL. Polypharmacy and medication management in older adults. Nurs Clin North Am. 2017:52(3):457-468.

[4] Abolbashari M, Macaulay TE, Whayne TF, Mukherjee D, Saha S. Polypharmacy in cardiovascular medicine: problems and promises! Cardiovasc Hematol Agents Med Chem. 2017;15(1):31-39.

Disclosure of Interests: Ana Paula Gomides Grant/research support from: Has received personal support and consulting fees from Pfizer and Janssen, Consultant for: Has received personal support and consulting fees from Pfizer and Janssen, Paid instructor for: Has received personal support and consulting fees from Pfizer and Janssen, Cleandro Albuquerque 